Research Paper

\title{
Cucurbitacin E inhibits cellular proliferation and enhances the chemo-response in gastric cancer by suppressing AKt activation
}

\author{
Wenzhang $\mathrm{Si}^{1^{*}}$, Jia Lyu ${ }^{2 *}$, Zhengchuang Liu ${ }^{3}$, Chunyang Wang ${ }^{4}$, Jingjing Huang ${ }^{4}$, Liping Jiang ${ }^{3}$, Tonghui \\ $\mathrm{Ma}^{3}$ \\ 1. Department of General Surgery, Affiliated Hospital of Shaoxing University (Shaoxing Municipal Hospital), Shaoxing, Zhejiang Province, China. \\ 2. Department of Urology, Zhejiang Provincial People's Hospital, Hangzhou Medical College, Hangzhou, Zhejiang 310014, China \\ 3. Key Laboratory of Gastroenterology of Zhejiang Province, Zhejiang Provincial People's Hospital, Hangzhou Medical College, Hangzhou, Zhejiang Province \\ 310014, China \\ 4. Genetron Health (Hangzhou) Medical Laboratory Co. Ltd, Hangzhou 310000, China \\ *These authors contributed equally to this work. \\ $\triangle$ Corresponding author: tonghuima0818@sina.com, and +86 0571 56509193; jianglp12@mails.jlu.edu.cn \\ (c) The author(s). This is an open access article distributed under the terms of the Creative Commons Attribution License (https://creativecommons.org/licenses/by/4.0/). \\ See http://ivyspring.com/terms for full terms and conditions.
}

Received: 2019.06.09; Accepted: 2019.08.20; Published: 2019.10.06

\begin{abstract}
Background: The incidence and mortality rate of gastric cancer has markedly declined over the past few decades, due to the progress and advances in the development of diagnostic and treatment regimens. However, there is still a large portion of patients who are first diagnosed during the advanced stage of gastric cancer when chemotherapy is needed. Unfortunately, resistance to chemotherapeutic agents is the most frequent occurrence during treatment, which indicates a need for the discovery of novel therapeutic anticancer drugs. Methods: The tumor-suppression effect of eight different cucurbitacins was evaluated in gastric cancer cell lines, and the Cucurbitacin E (CuE) showing the greatest effect was used in further studies to explore the mechanism and potential synergistic effect of Dox both in vitro and in vivo. Results: Compared with other cucurbitacins, CuE showed the greatest antiproliferative activity against the gastric cancer cell lines. Further investigations revealed that $\mathrm{CuE}$ suppressed the growth of gastric cancer cell lines through the induction of G2/M arrest and subsequent apoptosis by impairing AKt activation and reducing its expression in gastric cancer cells. Furthermore, our results indicate that $\mathrm{CuE}$ can significantly enhance the cytotoxicity of doxorubicin (Dox) both in vitro and in vivo. Conclusion: In summary, we present the first evidence of the efficacy of $\mathrm{CuE}$ for the inhibition of gastric cancer growth and the synergistic antitumorigenic effect of CuE and Dox, both in vitro and in vivo.
\end{abstract}

Key words: gastric cancer, Cucurbitacin E, doxorubicin, AKt

\section{Introduction}

Gastric cancer is the fourth most prevalent cancer[1]. It is also reportedly the second most lethal cancer worldwide, despite significant progress in detection and therapeutic regimens over the past decade. There is still a significant proportion of patients who receive the first diagnosis during the advanced gastric cancer, and hence, their prognosis is always associated with a poor outcome. The 5-year survival rate of these patients is only approximately
$31 \%$ in the United States (stage IA $94 \%$, stage IB $88 \%$, stage IIA $82 \%$, stage IIB $68 \%$, stage IIIA $54 \%$, stage IIIB $36 \%$, stage IIIC $18 \%$ )[2-4]. Patients diagnosed at the advanced stage had a 5 -year relative survival rate of $30 \%$ for males and $32 \%$ for females during the period 2002-2003 in Shanghai, China[5]. In practice, chemotherapy is more frequently administered to advanced-stage patients who have greater chances of benefitting from the treatment[6]. However, the 
resistance that develops during chemotherapy is a major obstacle for successful gastric cancer treatment. Most metastatic gastric cancers eventually develop resistance to chemotherapy, ultimately resulting in poor response rates[7]. Therefore, understanding the mechanisms by which tumors develop resistance to various agents is the key to identifying new genetic targets and combination therapies to fight resistance.

Recently, natural cucurbitacin compounds, which are tetracycline triterpene compounds derived from plants of the Cucurbitaceae family, have been shown to have effective pharmacological properties, such as anti-inflammatory and anticancer properties[8-12]. Cucurbitacin E (CuE), a cucurbitacin, has been shown to have remarkable potential in suppressing the proliferation of multiple cancer cell types[13]. Feng et al. demonstrated that CuE inhibits cancer cell progression via the inhibition of Wnt/ $\beta$-catenin signaling, and Wang's results suggest that $\mathrm{CuE}$ suppresses cytokine expression in human Jurkat $\mathrm{T}$ cells by inhibiting the activation of NF- $\mathrm{kB}[13$, 14]. The anticancer effects of cucurbitacins on diverse tumor types, such as breast cancer, neuroblastoma, lung cancer, endometrial cancer, and hepatocellular carcinoma, have been well studied. However, the efficacy of cucurbitacins for the suppression of gastric cancer growth has not been explored thus far[15-20].

The present study sought to assess the antitumorigenic effects of different cucurbitacins on gastric cancer cells. Thereafter, we selected $\mathrm{CuE}$, which was the most effective against gastric cancer cells for further study. In addition, $\mathrm{CuE}$ was administered to the xenografts to evaluate the antitumor activity in vivo. Then, we compared the tumor-suppressing efficacy of $\mathrm{CuE}$ alone and in combination with dox and investigated its potential anticancer mechanism. This study may be of significant functional relevance for exploration of cancer cell resistance to chemotherapeutic agents.

\section{Methods and materials}

\section{Cell lines and compounds used}

Human gastric cancer cell lines (NCI-N87, BGC-823, SNU-16, SGC-7901, and MGC-803) were purchased from the American Type Culture Collection (ATCC). Cucurbitacin A, B, D, S, IIa, and $\mathrm{IIb}(\mathrm{CuA}, \mathrm{CuB}, \mathrm{CuD}, \mathrm{CuS}, \mathrm{CuIIa}$ and $\mathrm{CuIIb})$ were obtained from ChemFaces (Wuhan, China). Cucurbitacin $\mathrm{E}$ and $\mathrm{I}(\mathrm{CuE}$ and $\mathrm{CuI})$ were from Sigma Aldrich (St. Louis, MO). The Cucurbitacins were dissolved using dimethyl sulfoxide (DMSO). Antibodies of PTEN, AKt, P-AKt and GADPH were procured from Cell Signaling Technology (Danvers, MA).

\section{Cell culture and treatments performed}

Human gastric cancer cell lines (NCI-N87, BGC-823, SNU-16, SGC-7901, and MGC-803) were passaged in RPMI-1640 medium supplemented with 10\% FBS (Invitrogen), $50 \mathrm{U} / \mathrm{ml}$ penicillin and 50 $\mu \mathrm{g} / \mathrm{ml}$ streptomycin, at a temperature of $37^{\circ} \mathrm{C}$ in the presence of $5 \% \mathrm{CO}_{2}$. Cellular growth was monitored using an inverted microscope. At $70-80 \%$ confluence, the cells were passaged using $0.25 \%$ trypsin. The cells were passaged every 3 to 4 days, and those in the logarithmic growth phase were used in experiments.

\section{Cellular viability}

The efficacy of the indicated compounds on gastric cancer proliferation was evaluated using a Cell Counting Kit-8 (CCK8, Sigma, St. Louis, MO). In brief, cells in the logarithmic growth phase were left to adhere on 96-well microplates at a density of $1 \times 10^{3}$ cells $/ \mathrm{ml}$ in $200 \mu \mathrm{l}$ of media containing $10 \%$ FBS. Twenty-four hours later, these cells were exposed to $10 \mu \mathrm{M}$ solutions of different cucurbitacins or DMSO for $48 \mathrm{~h}$, followed by the addition of $10 \mu \mathrm{l}$ of CCK8. The absorbance at $450 \mathrm{~nm}$ was detected postincubation at $37^{\circ} \mathrm{C}$ for $4 \mathrm{~h}$, using a microplate reader (Bio-Tek, USA). The results were analyzed using GraphPad Prism 5, and we multiplied the ratio of absorbance of drug-treated cells with that of untreated cells and multiplied the value by 100 to obtain the percentage of cell viability.

\section{Apoptosis assay}

Apoptosis was analyzed using a double-staining apoptosis detection kit with Annexin V-FITC and Propidium Iodide (PI) (eBioscience, San Diego, CA, USA). In brief, the NCI-N87 cells were treated with $\mathrm{CuE}(100 \mathrm{nM})$ or DMSO for $24 \mathrm{~h}$. Cells were collected using $0.25 \%$ trypsin and centrifuged at $300 \times \mathrm{g}$ for 5 min at room temperature, followed by washing twice in PBS. The cells were resuspended in $100 \mu \mathrm{l}$ of an appropriate binding buffer and stained using Annexin V/PI. The cells were enumerated using BD FACSDiva software (Becton Dickinson) and the BD FACS Canto II system flow cytometry platform.

\section{Cell cycle analysis}

The NCI-N87 cells were treated with CuE (100 $\mathrm{nM})$ or DMSO for $24 \mathrm{~h}$. A total of $5 \times 10^{5}$ cells were harvested, washed twice with PBS, and then fixed overnight with $70 \%$ methanol at $4^{\circ} \mathrm{C}$. The fixed cells were incubated with PI at $37^{\circ} \mathrm{C}$ for $30 \mathrm{~min}$ in the dark and then analyzed using a flow cytometer (BD FACS Canto II, Becton Dickinson).

\section{Western blotting}

The NCI-N87 cells were treated with CuE (25 
$\mathrm{nM}, 50 \mathrm{nM}, 100 \mathrm{nM}$ or $200 \mathrm{nM}$ ) or DMSO for $48 \mathrm{~h}$. Cellular lysis was carried out in lysis buffer containing protease inhibitor cocktail Z (Roche Applied Science, Germany). Equal amounts of whole protein extracts were resolved using SDS-PAGE and blotted onto PVDF membranes, which were then incubated with diluted primary antibodies and secondary antibodies. The analysis was performed using an ECL western blotting analysis system (Bio-Rad, CA, USA). ImageJ software was used for densitometric analyses.

\section{In vivo xenografts}

Female BALB/c nude mice (SLAC Laboratory Anim al, Shanghai, China) were housed at our institution and all animal experiments were conducted in accordance with institutional guidelines. Female BALB/c nude mice (4-5 weeks old) were subcutaneously injected with $1 \times 10^{7}$ NCI-N87 cells (suspended in $100 \mu \mathrm{l}$ of PBS with 50\% Matrigel (BD Biosciences)) to establish tumors. We used calipers to measure tumor dimensions. The formula: length $\times$ width $\times$ height $\times 0.5236$ was used for tumor volume enumeration[21]. Two weeks later, the tumor-bearing mice were segregated into four groups of 6 per group, and all groups were administered an intraperitoneal injection of either (i) $0.15 \mathrm{ml}$ of PBS every 3 days, (ii) $0.30 \mathrm{mg} / \mathrm{kg}$ of CuE every 3 days, (iii) $2 \mathrm{mg} / \mathrm{kg}$ of Dox every 3 days, or (iv) $0.30 \mathrm{mg} / \mathrm{kg}$ of $\mathrm{CuE}$ and $2 \mathrm{mg} / \mathrm{kg}$ of Dox every 3 days[22]. We used the formula volume $=$ width $^{2} \times$ length $/ 2$ to calculate the tumor volume (in $\mathrm{mm}^{3}$ ), and plotted a tumor growth curve. The tumors were monitored until the mice were sacrificed.

\section{Statistical analysis}

We performed all experiments in triplicate, unless otherwise noted, and the data are presented as the mean \pm S.D. GraphPad Prism software was used for calculations, and a $p$ value of less than 0.05 was considered indicative of statistical significance.

\section{Results}

\section{Cucurbitacins have a cytotoxic effect on cultured gastric cancer cells}

Eight cucurbitacins $(\mathrm{CuA}, \mathrm{CuB}, \mathrm{CuD}, \mathrm{CuE}, \mathrm{CuI}$, $\mathrm{CuS}$, CuIla, CuIIb) were incubated with five gastric cancer cell lines (NCI-N87, BGC-823, SNU-16, SGC-7901, and MGC-803) at a concentration of $10 \mu \mathrm{M}$ for $48 \mathrm{~h}$, to study the toxicity of cucurbitacins on gastric cancer cells. We measured cellular viability using a CCK8 assay. All cucurbitacins exhibited antitumor efficacy on gastric cancer growth, compared with that of DMSO. The antiproliferative effect of $\mathrm{CuB}, \mathrm{CuD}, \mathrm{CuE}$ and $\mathrm{CuI}$ was found to be more pronounced than that of the other cucurbitacins, while $\mathrm{CuE}$ showed the highest potential out of all the cucurbitacins, by killing more than $70 \%$ of cells (Fig. 1A). In order to further investigate how $\mathrm{CuE}$ affects gastric cancer, all five cell lines mentioned above were treated with varying concentrations (from $0 \mathrm{nM}$ to 300 $\mathrm{nM})$ of CuE. CuE exhibited dose dependent cytotoxicity on gastric cancer cells and its $\mathrm{IC}_{50}$ (half maximal inhibitory concentration) ranged from $80 \mathrm{nM}$ to $130 \mathrm{nM}$ (Fig. 1B).

In order to further confirm that $\mathrm{CuE}$ induces apoptosis, the NCI-N87 cell line was treated with CuE $(100 \mathrm{nM})$, and DMSO was used as the control. After 24 $\mathrm{h}$ of incubation, Annexin V/PI staining of the cells revealed a higher percentage of apoptotic cells upon $\mathrm{CuE}$ treatment than upon DMSO treatment (Fig. 2A and $2 \mathrm{~B}$ ).

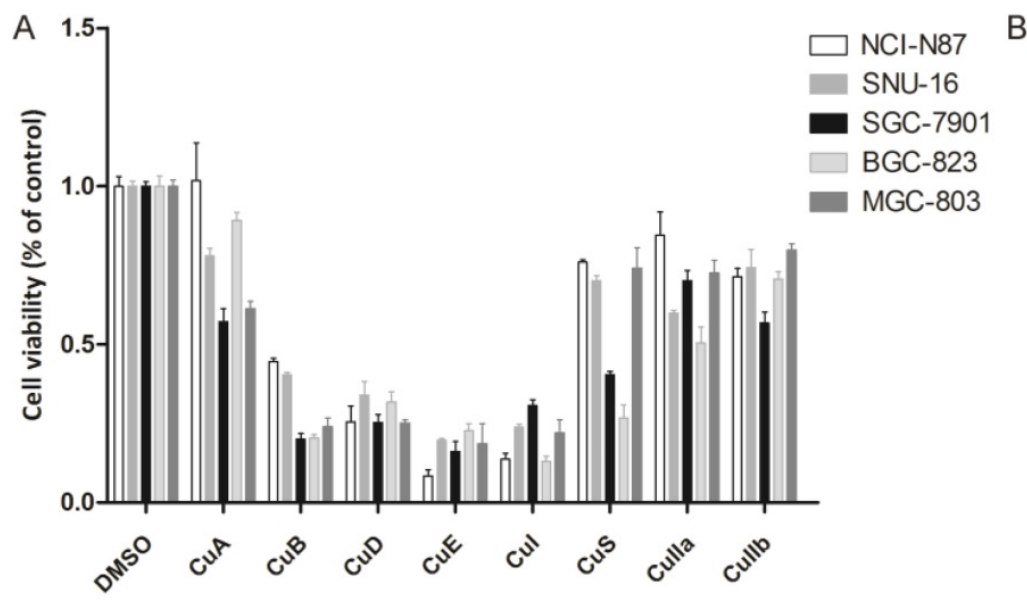

B

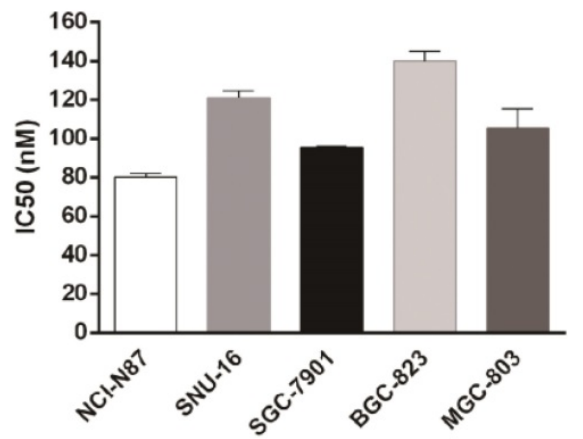

Figure 1. CuE effectively inhibits gastric cell proliferation. Cells (NCI-N87, SNU-16, MGC-803, SGC-7901, and BGC-823) were treated with various cucurbitacins as indicated. (A) Cell viability was estimated using CCK8 assays. (B) Cells were treated with different doses of CuE (from $0 \mathrm{nM}$ to $300 \mathrm{nM}$ ), analyzed using the CCK8 assay, and the $I_{50}$ was measured. 
A

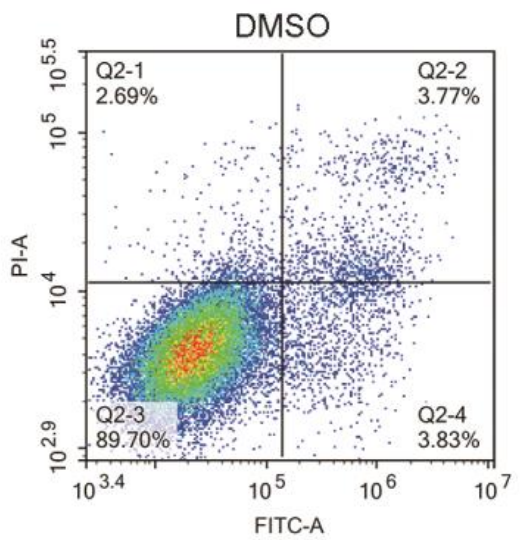

B

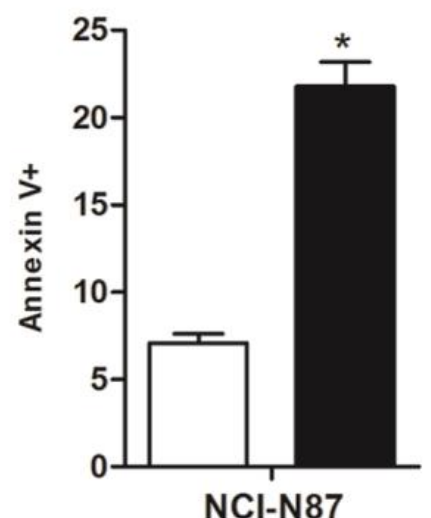

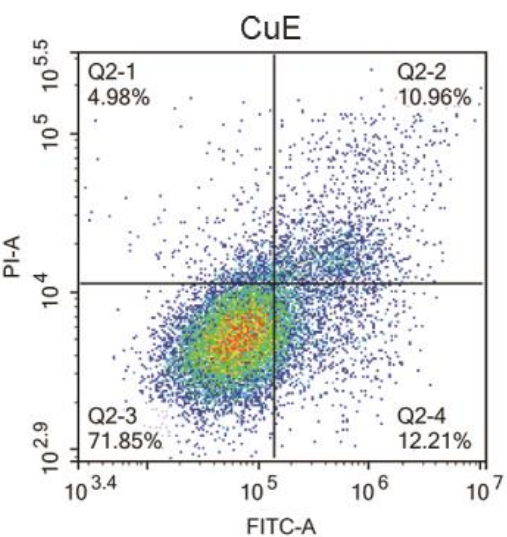

DMSO

CuE

Finduces apoptosis of $\mathbf{N C l}-\mathbf{N} 87$ cells. $\mathrm{NCl}-\mathrm{N} 87$ cells were exposed to CuE (100 nM) or DMSO for $24 \mathrm{~h}$, and staining. (A) The effects of CuE on apoptosis assessed using flow cytometry are presented. (B) The percentage of Annexin $\mathrm{V}+$ cells, which represent apoptotic cells, is shown $(p=0.0014)$.

\section{CuE causes changes in cell cycle distribution by suppressing the activation of AKt}

The NCI-N87 cells were incubated with CuE (100 $\mathrm{nM}$ ) or DMSO for $24 \mathrm{~h}$ to explore the inhibitory mechanism of $\mathrm{CuE}$. Flow cytometric analysis of the cell cycle showed that $\mathrm{CuE}$ treatment caused G2/M arrest and significantly increased the proportion of cells that were at the G2/M phase (Fig. 3A and 3B).

Previous studies have suggested that preventing the activation of AKt induces G2/M arrest in diverse types of cancer cells, such as breast, lung and prostate cancer cells[23-25]. CuE has been suggested to inhibit the activation of AKt and enhance PTEN expression in lung cancer cells[26]. Therefore, the effect of $\mathrm{CuE}$ on AKt in gastric cancer cells was studied. After incubation with varying concentrations of $\mathrm{CuE}$, we found a dose-dependent decrease in both pAKt and AKt levels, as shown by western blotting analysis. This observation implies that CuE suppresses the activation of $\mathrm{AKt}$ in gastric cancer cells in a dose-dependent manner, and inhibits the expression of AKt but shows no effect on PTEN (Fig. 3C). Then, we analyzed AKt mRNA levels in gastric cancer tumors and normal tissue using data from The Cancer Genome Atlas (TCGA) and found that AKt mRNA levels were higher in gastric tumors than in normal tissue (Fig. 4A). In addition, IHC detection of AKt and pAKt was performed on 4 pairs of gastric cancer and normal tissue specimens obtained from cancer patients. The relative protein expression intensity of $\mathrm{AKt}$ and pAKt was higher in gastric cancer tissues than in normal tissues (Fig. 4B).

\section{Suppression of $\mathrm{AKt}$ activation by $\mathrm{CuE}$ potentiates the cytotoxicity of Dox in NCI-N87 cells}

Recently, several studies have demonstrated the synergistic effects of AKt inhibitors in combination with Dox. Babichev found that inhibiting the $\mathrm{PI} 3 \mathrm{~K} / \mathrm{AKt} / \mathrm{mTOR}$ pathway could significantly enhance the effect of Dox on LMS cell lines, and the tumor volume of the group treated with the AKt inhibitor BEZ235 in combination with Dox decreased by $71 \%$ in comparison with that of the vehicle-treated group[27]. $\mathrm{Hu}$ and colleagues revealed that NVP-BKM120 (BKM120), a selective inhibitor of PI3K, also suppresses the activation of $\mathrm{AKt}$ and that it shows a strong synergistic antiproliferative effect in multidrug resistant (MDR) breast cancer cells, when combined with Dox[28]. Since CuE inhibits AKt activation, we explored whether $\mathrm{CuE}$ could enhance 
the cytotoxicity of Dox in gastric cancer cells. As expected, compared with Dox treatment alone, the combined CuE $(60 \mathrm{nM})$ treatment significantly increased the sensitivity of NCI-N87 gastric cancer cells to Dox (IC50: $100 \mathrm{nM}$ vs. $700 \mathrm{nM}$ ) (Fig. 5A). To further assess whether CuE/Dox-induces cell death is due to apoptosis, Annexin V/PI staining assay was performed and quantified by flow cytometry. As illustrated in Fig. $5 \mathrm{~B}$ and $5 \mathrm{C}$, compared to the Dox-only treated group, the $\mathrm{CuE} /$ Dox-treated group showed a significantly increased percentage of Annexin V-positive apoptotic cells (40\% vs. $80 \%$ ). These results demonstrate the potential of $\mathrm{CuE}$ to increase the cytotoxicity of Dox in NCI-N87 gastric cancer cells by promoting apoptosis.

\section{Synergistic growth inhibition of gastric cancer xenografts by CuE and Dox}

It has previously been observed that $\mathrm{CuE}$

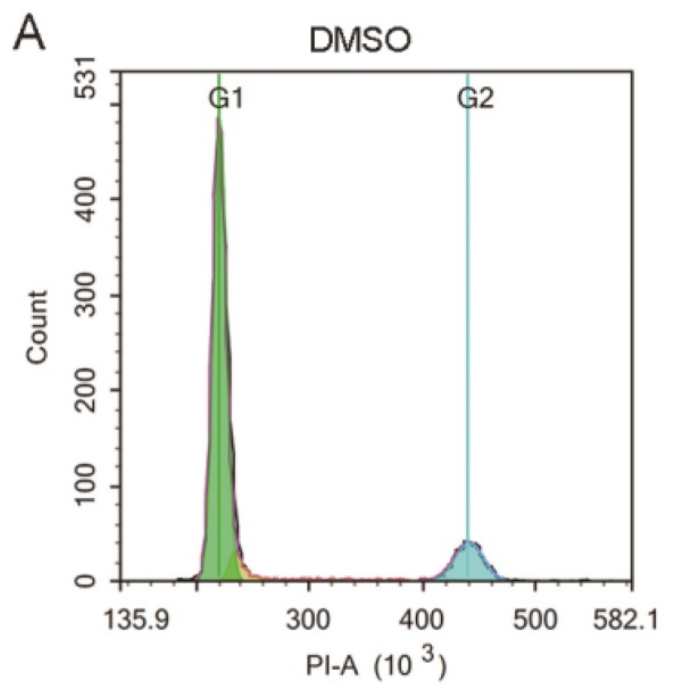

B

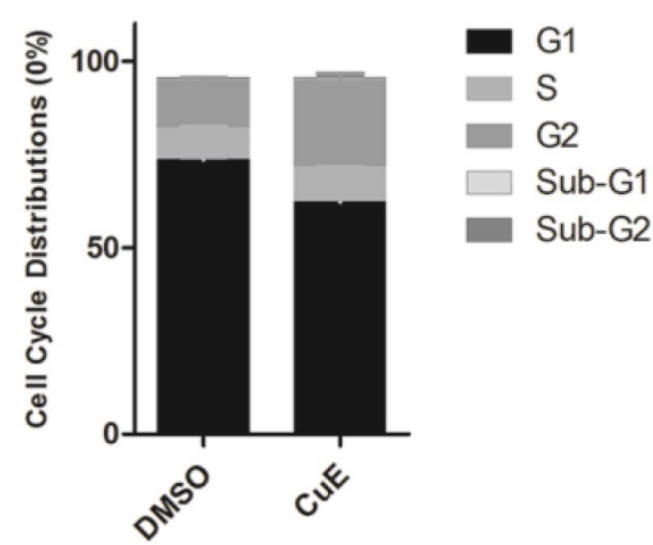

inhibits the proliferation of gastric NCI-N87 cancer cells and that it enhances the sensitivity of NCI-N87 cells to Dox in vitro. We evaluated the effects of $\mathrm{CuE}$ and Dox monotherapy, as well as the combination therapy on the growth of NCI-N87 xenografts. BALB/c mice bearing NCI-N87 xenografts were arbitrarily divided into 4 groups and injected intraperitoneally with DMSO (placebo), CuE, Dox, or the combination of $\mathrm{CuE}$ and Dox for a period of 4 weeks (Fig. 6A). The animals were sacrificed, and tumor volumes were estimated 40 days post establishment of the xenografts. Consistent with the in vitro results, the combination group showed significantly lower volumes of xenograft tumors than the $\mathrm{CuE}$ and Dox monotherapy groups, which indicates that combination therapy is more effective in abrogating cellular proliferation than the vehicle or either monotherapy (Fig. 6B).

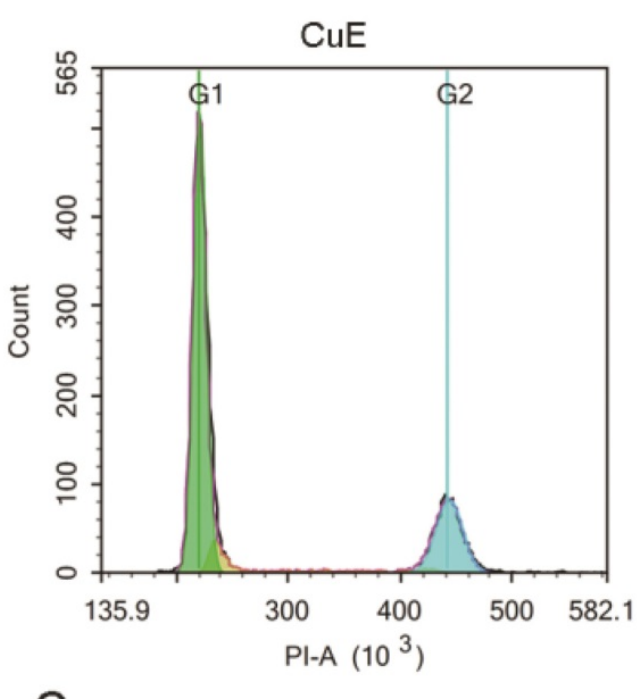

C

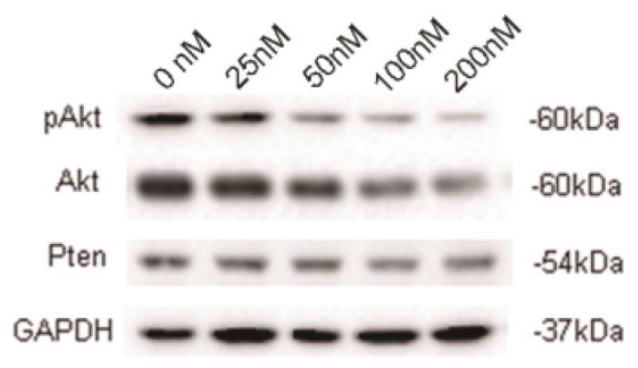

CuE treatment

Figure 3. CuE induces G2/M arrest and inhibits AKt activation of NCI-N87 cells. (A) NCl-N87 cells were exposed to $100 \mathrm{nM}$ CuE or DMSO for 24 h, followed by PI staining and cell cycle profiling using flow cytometric analysis. The horizontal and vertical axes represent the intensity of $\mathrm{PI}$ staining and cell counts, respectively. (B) $\mathrm{Quantitative}$ data of cell cycle distribution are shown in panel A. (C) NCl-N87 cells were cultured in the presence of DMSO or increasing concentrations of CuE ( $25 \mathrm{nM}, 50 \mathrm{nM}, 100 \mathrm{nM}$ or $200 \mathrm{nM}$ ) for $48 \mathrm{~h}$, and the cells were lysed for western blotting analysis. 

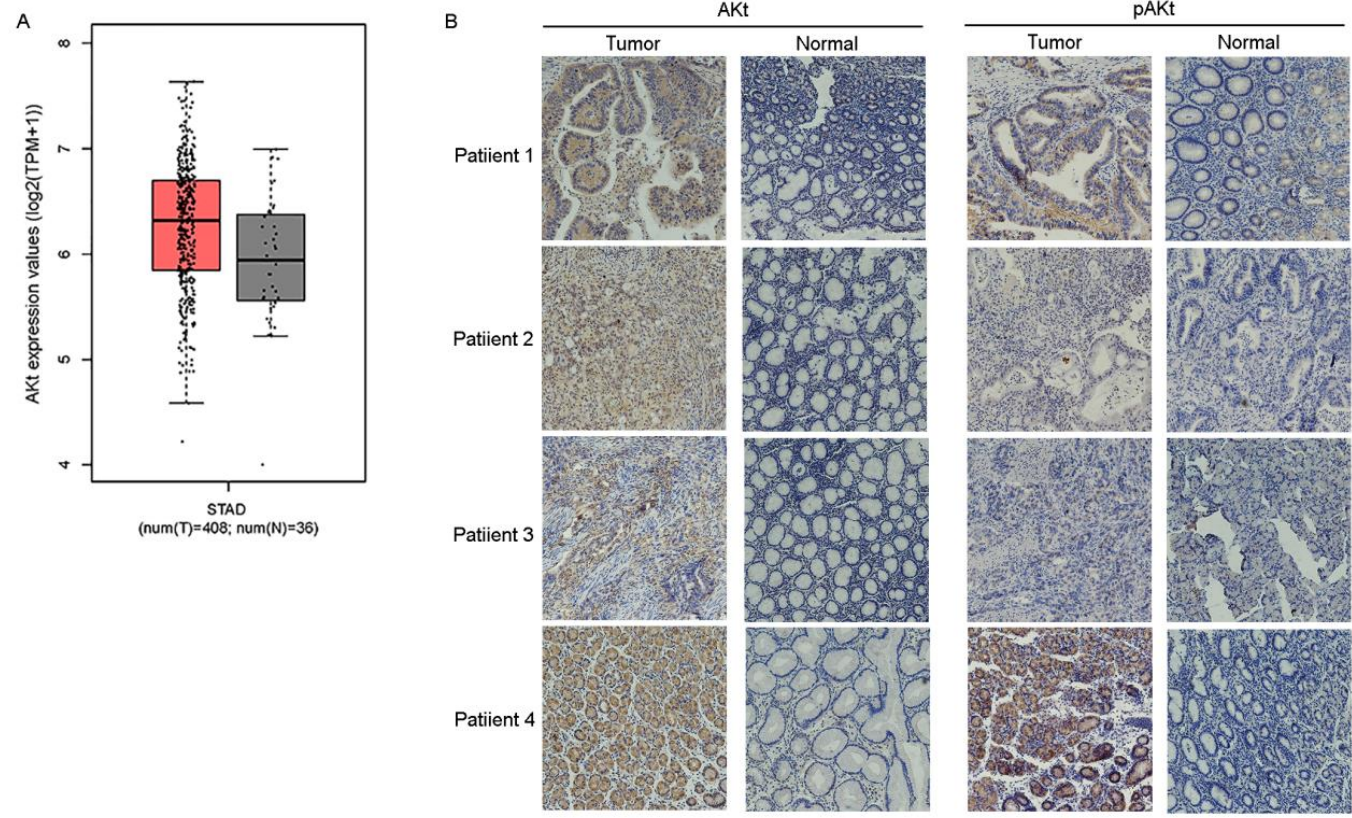

Figure 4. AKt mRNA levels was higher in gastric tumors than in normal tissue in IHC assay. Analysis of AKtl mRNA levels in gastric cancer tumors and normal tissue from TCGA data (A) and immunohistochemical analysis of the expression levels of Akt and pAKt in 4 pairs of gastric cancer and normal tissue from cancer patients (B).

A

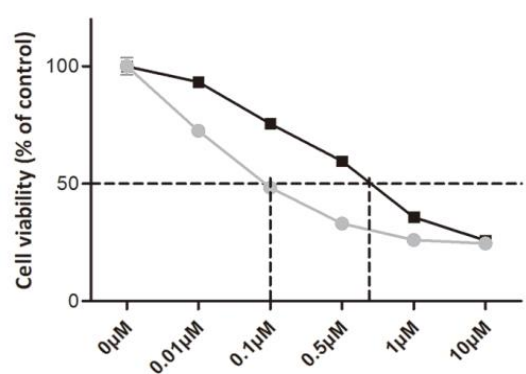

C
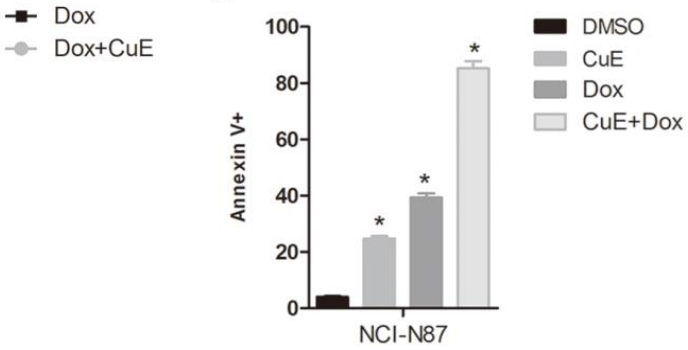

B
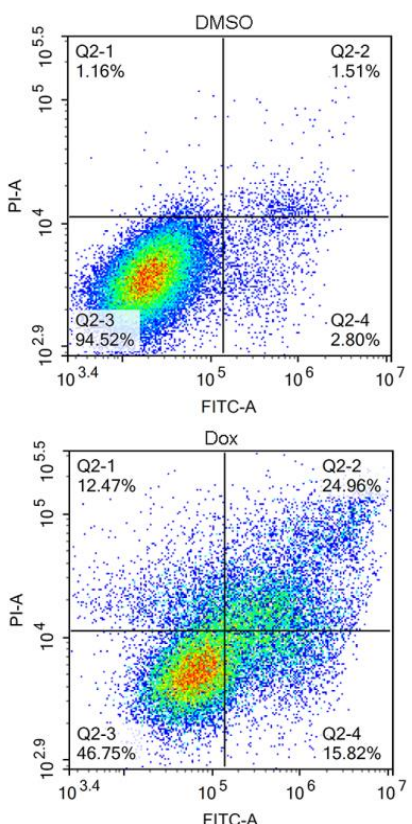

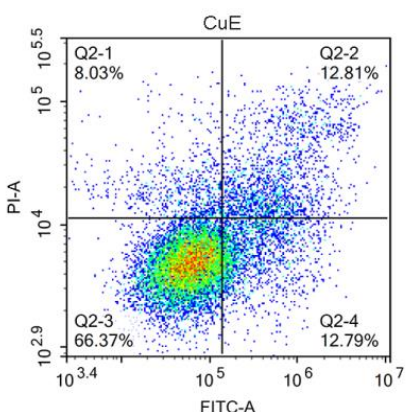

CuE+Dox

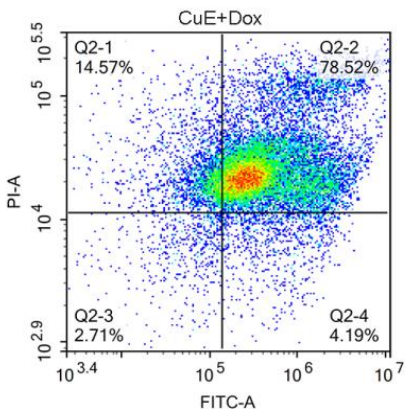

Figure 5. The suppression of AKt activation by $\mathrm{CuE}$ potentiates Dox-induced cytotoxicity in $\mathrm{NCI}-\mathrm{N} 87$ cells. NCl-N87 cells were treated with Dox $(0 \mu \mathrm{M}, 0.01$ $\mu \mathrm{M}, 0.1 \mu \mathrm{M}, 0.5 \mu \mathrm{M}, 1 \mu \mathrm{M}$ or $10 \mu \mathrm{M})$ or a combination of CuE $(60 \mathrm{nM})$ plus Dox $(0 \mu \mathrm{M}, 0.01 \mu \mathrm{M}, 0.1 \mu \mathrm{M}, 0.5 \mu \mathrm{M}, 1 \mu \mathrm{M}$ or $10 \mu \mathrm{M})$ for $48 \mathrm{~h}$. (A) A CCK8 assay was used to measure cell viability. (B) NCl-N87 cells were treated with DMSO, Dox (500 nM), CuE (60 nM) or a combination of Dox (500 nM) plus CuE (60 nM) for $48 \mathrm{~h}$, subjected to Annexin V/PI staining, and analyzed by flow cytometry. (C) The percentage of Annexin V-positive cells representing apoptotic cells is shown (CuE+Dox vs. Dox, $\mathrm{P}=0.0044)$. 
A

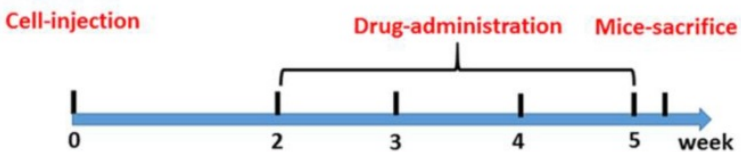

B
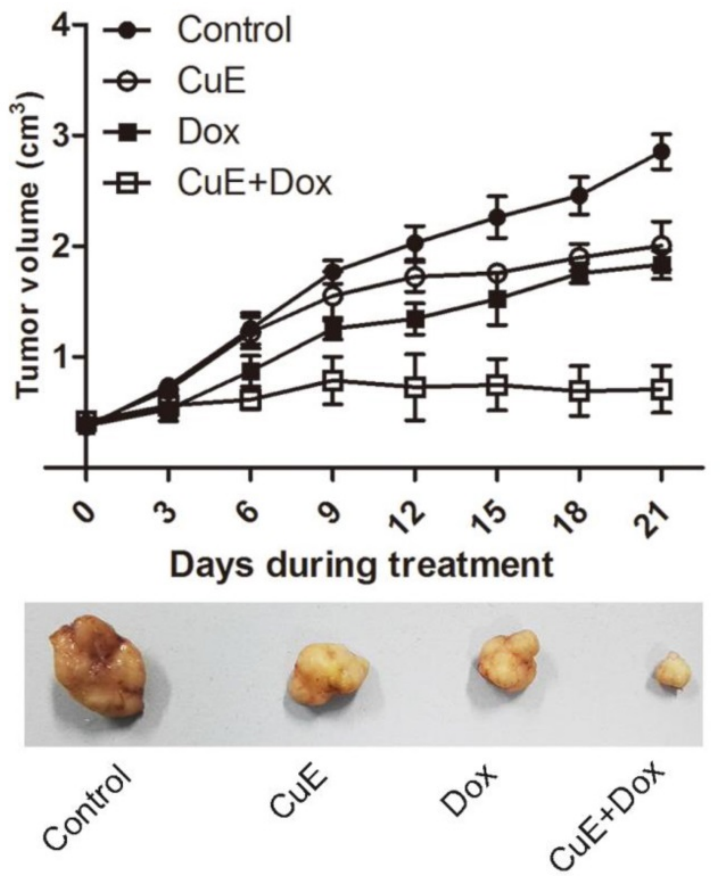

Figure 6. The synergistic inhibition of gastric xenografts by CuE and Dox. Four- to five-week-old female nude mice were injected on their right flank with $1 \times 10^{7}$ $\mathrm{NCl}-\mathrm{N} 87$ cells suspended in $100 \mu \mathrm{l}$ of PBS with $50 \%$ Matrigel. One week later, the mice were randomly segregated into four treatment groups: control group (DMSO), CuE group ( $0.30 \mathrm{mg} / \mathrm{kg}$, every 3 days), Dox group ( $2 \mathrm{mg} / \mathrm{kg}$, every 3 days), CuE plus Dox group $(0.30 \mathrm{mg} / \mathrm{kg}+2 \mathrm{mg} / \mathrm{kg}$, every 3 days). (A) The experimental strategy is shown. (B) Tumor diameters were measured every 3 days, until the mice were sacrificed, and a representative tumor from each group is shown.

\section{Discussion}

Although major advances have been made regarding gastric cancer treatments, patients with advanced stages of the disease still show a poor prognosis. Unfortunately, the OS of gastric cancer patients receiving systemic chemotherapy during the late stage is less than 1 year[29]. Chemoresistance in some forms of these cancers is still a significant challenge for the treatment of cancer and clearly requires more effective and safe regimens[30].

Research concerning combinatorial chemotherapy has gradually gained attention from researchers in the field of gastric cancer research. Additionally, several recent reports have demonstrated the inhibitory effects of cucurbitacins on human cancer cells and tumor xenografts through the inhibition of STAT3 phosphorylation[31, 32]. Cucurbitacins are components of plant-based medicines used in Asian countries, such as China and India. Cucurbitacins are known for their antiproliferative activity on diverse forms of cancers [33, 34].

This study investigated the effect of cucurbitacins on gastric cancer proliferation and elucidated the underlying mechanism of action. All eight cucurbitacins used in the study produced an antitumor effect on gastric cancer growth when used on the five included gastric cancer cell lines. Despite recent reports that $\mathrm{CuI}$ inhibits gastric cancer cell growth, interestingly, we found that $\mathrm{CuE}$ possesses greater inhibitory potential than $\mathrm{CuI}$, the cause of which might be ascribed to diverse molecular mechanisms in different gastric cancer cell lines[35].

We explored the inhibitory mechanisms of $\mathrm{CuE}$ on gastric cancer through the apoptosis and the cell cycle distribution of CuE- or DMSO-treated NCI-N87 cells. CuE treatment induced significant levels of apoptosis in comparison with the placebo treatment. Further investigations revealed that $\mathrm{CuE}$ induced G2/M arrest in NCI-N87 cells (Fig. 3A and 3B). We noted that previous work revealed the importance of the activation state of AKt in cellular apoptosis. Kim and colleagues demonstrated that activation of the PI3-K/AKt pathway reduces ASK1-induced apoptosis[36]. In contrast, Frias et al. found that AKt inhibitors (AKti-1/2 and A-443654) induce the apoptosis of chronic lymphocytic leukemia cells in a dose-dependent manner[37]. In addition to playing a key role in apoptosis, AKt is also required for the transition of cells into the G2/M phase[38]. Treatment of avian embryo retinas with a PI3K inhibitor (LY 294002) induced G2/M inhibition in late progenitors, which was mediated through the suppression of phospho-AKt[39]. Therefore, we evaluated the function of $\mathrm{AKt}$ in $\mathrm{CuE}$-mediated inhibition of gastric cancer and found that $\mathrm{CuE}$ inhibits phospho-AKt in a dose-dependent manner. Interestingly, AKt levels also decreased along with the concomitant increase in the concentration of $\mathrm{CuE}$, which was hitherto unknown. It has been previously reported that $\mathrm{CuB}$, a member of the cucurbitacin family, upregulates PTEN expression and thereby affects the subsequent activation of $\mathrm{AKt}$; we also found that $\mathrm{CuE}$ has little effect on PTEN expression (Fig. 3C).

Previous studies have suggested that the activation of $\mathrm{PI} 3 \mathrm{~K} / \mathrm{AKt}$ signaling is frequently involved in the development of resistance to chemotherapy[40, 41]. Once activated, AKt activates multiple substrates and subsequent effectors to promote cell survival, proliferation and chemoresistance. Suppression of AKt activation by PI3K or AKt inhibitors has been suggested to be a valid approach for treating cancer and increasing the efficacy of chemotherapy[42]. In this study, we found that $\mathrm{CuE}$ treatment clearly inhibited AKt activation 
and boosted the cytotoxicity of chemotherapeutic agents such as Dox in vitro and in vivo, which indicates a clear synergistic effect with Dox in gastric cancer.

Chemotherapy is an important treatment option for advanced gastric cancers. However, the frequent occurrence of resistance to chemotherapy impairs the response rates of patients. Various efforts, such as combinatorial drug therapy, have been used to overcome chemoresistance. Our present study demonstrates the efficacy of $\mathrm{CuE}$ in different cancer cells of gastric origin. We have also identified, for the first time, the role $\mathrm{CuE}$ plays in the inhibition of gastric tumor growth by targeting the AKt signaling pathway. Furthermore, our results demonstrate that $\mathrm{CuE}$ displays the potential to enhance gastric cancer sensitivity to Dox-dependent treatment regimens. Our study provides a strong clinical rationale for the use of $\mathrm{CuE}$ as a novel pharmaceutical chemosensitizer for the treatment of gastric cancer.

\section{Acknowledgments}

All authors declare that they have no potential conflicts of interest in the study. Informed consent was obtained from all individual participants included in the study. This work was supported by the Shaoxing science and technology project (Grant No. 2017B70050), the Zhejiang Province medical and health research projects (Grant No. 2016KYB027 and Grant No. 2017KY010).

\section{Competing Interests}

The authors have declared that no competing interest exists.

\section{References}

1. Majeed W, Iftikhar A, Khaliq T et al. Gastric Carcinoma: Recent Trends in Diagnostic Biomarkers and Molecular Targeted Therapies. Asian Pac J Cancer Prev 2016; 17: 3053-3060.

2. Siegel R, Naishadham D, Jemal A. Cancer statistics, 2012. CA Cancer J Clin 2012; 62: 10-29.

3. Jemal A, Bray F, Center MM et al. Global cancer statistics. CA Cancer J Clin 2011; 61: 69-90.

4. Ferlay J, Soerjomataram I, Dikshit R et al. Cancer incidence and mortality worldwide: sources, methods and major patterns in GLOBOCAN 2012. Int J Cancer 2015; 136: E359-386.

5. Zheng $\mathrm{L}, \mathrm{Wu} \mathrm{C}, \mathrm{Xi} \mathrm{P}$ et al. The survival and the long-term trends of patients with gastric cancer in Shanghai, China. BMC Cancer 2014; 14: 300.

6. Lordick F, Lorenzen S, Yamada Y, Ilson D. Optimal chemotherapy for advanced gastric cancer: is there a global consensus? Gastric Cancer 2014; 17: 213-225

7. Cho SJ, Park JW, Kang JS et al. Nuclear factor-kappaB dependency of doxorubicin sensitivity in gastric cancer cells is determined by manganese superoxide dismutase expression. Cancer Sci 2008; 99: 1117-1124.

8. Chen JC, Chiu MH, Nie RL et al. Cucurbitacins and cucurbitane glycosides: structures and biological activities. Nat Prod Rep 2005; 22: 386-399.

9. Song $\mathrm{Y}$, Ding $\mathrm{N}$, Kanazawa $\mathrm{T}$ et al. Cucurbitacin $\mathrm{D}$ is a new inflammasome activator in macrophages. International Immunopharmacology 2013; 17: 1044.
10. Wang Y, Zhao GX, Xu LH et al. Cucurbitacin IIb exhibits anti-inflammatory activity through modulating multiple cellular behaviors of mouse lymphocytes. PLoS One 2014; 9: e89751.

11. Siqueira JM, Gazola AC, Farias MR et al. Evaluation of the antitumoral effect of dihydrocucurbitacin-B in both in vitro and in vivo models. Cancer Chemother Pharmacol 2009; 64: 529-538.

12. Escandell JM, Recio MC, Manez S et al. Dihydrocucurbitacin B inhibits delayed type hypersensitivity reactions by suppressing lymphocyte proliferation. J Pharmacol Exp Ther 2007; 322: 1261-1268.

13. Feng H, Zang L, Zhao ZX, Kan QC. Cucurbitacin-E inhibits multiple cancer cells proliferation through attenuation of Wnt/beta-catenin signaling. Cancer Biother Radiopharm 2014; 29: 210-214.

14. Wang L, Li C, Lin Q et al. Cucurbitacin E suppresses cytokine expression in human Jurkat $\mathrm{T}$ cells through down-regulating the NF-kappaB signaling. Acta Biochim Biophys Sin (Shanghai) 2015; 47: 459-465.

15. Kong Y, Chen J, Zhou Z et al. Cucurbitacin E induces cell cycle G2/M phase arrest and apoptosis in triple negative breast cancer. PLoS One 2014; 9: e103760.

16. Lan T, Wang L, Xu Q et al. Growth inhibitory effect of Cucurbitacin E on breast cancer cells. Int J Clin Exp Pathol 2013; 6: 1799-1805.

17. Shang Y, Guo XX, Li WW et al. Cucurbitacin-B inhibits neuroblastoma cell proliferation through up-regulation of PTEN. Eur Rev Med Pharmacol Sci 2014; 18: 3297-3303.

18. Jacquot C, Rousseau B, Carbonnelle D et al. Cucurbitacin-D-induced CDK1 mRNA up-regulation causes proliferation arrest of a non-small cell lung carcinoma cell line (NSCLC-N6). Anticancer Res 2014; 34: 4797-4806.

19. Ishii $\mathrm{T}$, Kira N, Yoshida $\mathrm{T}$, Narahara H. Cucurbitacin $\mathrm{D}$ induces growth inhibition, cell cycle arrest, and apoptosis in human endometrial and ovarian cancer cells. Tumour Biol 2013; 34: 285-291.

20. Liu K, Xing H, Zhang S et al. Cucurbitacin D induces fetal hemoglobin synthesis in K562 cells and human hematopoietic progenitors through activation of p38 pathway and stabilization of the gamma-globin mRNA. Blood Cells Mol Dis 2010; 45: 269-275.

21. Kiaris H, Schally AV, Varga JL. Antagonists of growth hormone-releasing hormone inhibit the growth of U-87MG human glioblastoma in nude mice. Neoplasia 2000; 2: 242-250.

22. Sinha S, Khan S, Shukla S et al. Cucurbitacin B inhibits breast cancer metastasis and angiogenesis through VEGF-mediated suppression of FAK/MMP-9 signaling axis. Int J Biochem Cell Biol 2016; 77: 41-56.

23. Zhao L, Miao H, Li W et al. LW - 213 induces G2/M cell cycle arrest through AKT/GSK3 $\beta / \beta$ - catenin signaling pathway in human breast cancer cells. Molecular Carcinogenesis 2015; 55: 778.

24. Xu X, Zhang Y, Qu D et al. Osthole induces G2/M arrest and apoptosis in lung cancer A549 cells by modulating PI3K/Akt pathway. Journal of Experimental \& Clinical Cancer Research 2011; 30: 33.

25. Yan X, Shen H, Jiang $H$ et al. External Qi of Yan Xin Qigong induces $\mathrm{G} 2 / \mathrm{M}$ arrest and apoptosis of androgen-independent prostate cancer cells by inhibiting Akt and NF-kB pathways. Molecular and Cellular Biochemistry 2007; 310: 227-234.

26. Ma G, Luo W, Lu J et al. Cucurbitacin E induces caspase-dependent apoptosis and protective autophagy mediated by ROS in lung cancer cells. Chemico-Biological Interactions 2016; 253: 1-9.

27. Babichev Y, Kabaroff L, Datti A et al. PI3K/AKT/mTOR inhibition in combination with doxorubicin is an effective therapy for leiomyosarcoma. J Transl Med 2016; 14: 67.

28. Hu Y, Guo R, Wei J et al. Effects of PI3K inhibitor NVP-BKM120 on overcoming drug resistance and eliminating cancer stem cells in human breast cancer cells. Cell Death and Disease 2015; 6 .

29. Wagner AD, Grothe W, Haerting J et al. Chemotherapy in advanced gastric cancer: a systematic review and meta-analysis based on aggregate data. J Clin Oncol 2006; 24: 2903-2909.

30. Nishikawa S, Konno M, Hamabe A et al. Aldehyde dehydrogenasehigh gastric cancer stem cells are resistant to chemotherapy. International Journal of Oncology 2013; 42: 1437.

31. Jayaprakasam B, Seeram NP, Nair MG. Anticancer and antiinflammatory activities of cucurbitacins from Cucurbita andreana. Cancer Lett 2003; 189: 11-16.

32. Sun J, Blaskovich MA, Jove R et al. Cucurbitacin Q: a selective STAT3 activation inhibitor with potent antitumor activity. Oncogene 2005; 24 : 3236-3245.

33. Liu $\mathrm{T}$, Zhang $\mathrm{M}$, Zhang $\mathrm{H}$ et al. Inhibitory effects of cucurbitacin B on laryngeal squamous cell carcinoma. Eur Arch Otorhinolaryngol 2008; 265: 1225-1232.

34. Tannin-Spitz T, Grossman S, Dovrat $\mathrm{S}$ et al. Growth inhibitory activity of cucurbitacin glucosides isolated from Citrullus colocynthis on human breast cancer cells. Biochem Pharmacol 2007; 73: 56-67.

35. Deng $C$, Zhang B, Zhang $S$ et al. Low nanomolar concentrations of Cucurbitacin-I induces G2/M phase arrest and apoptosis by perturbing 
redox homeostasis in gastric cancer cells in vitro and in vivo. Cell Death and Disease 2016; 7.

36. Kim AH, Khursigara G, Sun $\mathrm{X}$ et al. Akt phosphorylates and negatively regulates apoptosis signal-regulating kinase 1. Molecular and Cellular Biology 2001; 21: 893-901.

37. De Frias M, Iglesiasserret D, Cosialls AM et al. Akt inhibitors induce apoptosis in chronic lymphocytic leukemia cells. Haematologica-the Hematology Journal 2009; 94: 1698.

38. Lee S, Park J, Park EK et al. Akt - induced promotion of cell - cycle progression at $\mathrm{G} 2 / \mathrm{M}$ phase involves upregulation of $\mathrm{NF}-\mathrm{Y}$ binding activity in PC12 cells. Journal of Cellular Physiology 2005; 205: 270-277.

39. Ornelas IM, Silva TM, Fragelmadeira L, Ventura ALM. Inhibition of PI3K/Akt pathway impairs G2/M transition of cell cycle in late developing progenitors of the avian embryo retina. PLOS ONE 2013; 8.

40. West KA, Castillo SS, Dennis PA. Activation of the PI3K/Akt pathway and chemotherapeutic resistance. Drug Resistance Updates 2002; 5: 234-248.

41. Ghebeh H, Alkhaldi S, Olabi S et al. Fascin is involved in the chemotherapeutic resistance of breast cancer cells predominantly via the PI3K/Akt pathway. British Journal of Cancer 2014; 111: 1552.

42. Babichev Y, Kabaroff L, Datti A et al. PI3K/AKT/mTOR inhibition in combination with doxorubicin is an effective therapy for leiomyosarcoma. Journal of Translational Medicine 2016; 14: 1-12. 